PROCEEDINGS OF THE

AMERICAN MATHEMATICAL SOCIETY

Volume 134, Number 6, Pages 1599-1604

S 0002-9939(05)08170-0

Article electronically published on December 2, 2005

\title{
POINCARÉ DUALITY ALGEBRAS AND RINGS OF COINVARIANTS
}

\author{
TZU-CHUN LIN
}

(Communicated by Bernd Ulrich)

\begin{abstract}
Let $\varrho: G \hookrightarrow G L(n, \mathbb{F})$ be a faithful representation of a finite group $G$ over the field $\mathbb{F}$. Via $\varrho$ the group $G$ acts on $V=\mathbb{F}^{n}$ and hence on the algebra $\mathbb{F}[V]$ of homogenous polynomial functions on the vector space $V$. R. Kane (1994) formulated the following result based on the work of R. Steinberg (1964): If the field $\mathbb{F}$ has characteristic 0 , then $\mathbb{F}[V]_{G}$ is a Poincaré duality algebra if and only if $G$ is a pseudoreflection group. The purpose of this note is to extend this result to the case $|G| \in \mathbb{F}^{\times}$(i.e. the order $|G|$ of $G$ is relatively prime to the characteristic of $\mathbb{F}$ ).
\end{abstract}

\section{INTRODUCTION}

Let $G$ be a finite group, $\mathbb{F}$ a field and $V$ an $n$-dimensional $\mathbb{F}$-vector space. For a representation $\varrho: G \hookrightarrow G L(n, \mathbb{F}) \cong G L(V)$ the group $G$ acts on the algebra $\mathbb{F}[V]$ of homogeneous polynomial functions on $V=\mathbb{F}^{n}$. The ring of invariants is defined by

$$
\mathbb{F}[V]^{G}:=\{f \in \mathbb{F}[V] \mid \sigma f=f, \forall \sigma \in G\} .
$$

We define the ring of coinvariants to be the graded quotient algebra

$$
\mathbb{F}[V]_{G}:=\mathbb{F}[V] / h(G),
$$

where $h(G)$ is the ideal in $\mathbb{F}[V]$ generated by all $G$-invariant homogeneous polynomials of strictly positive degree. $h(G)$ is called the Hilbert's ideal. As a convenient reference for invariant theory see [2] or [14].

Note that the Hilbert's ideal $h(G)$ is a $\overline{\mathbb{F}[V]}$-primary ideal, where $\overline{\mathbb{F}[V]}$ denotes the augmentation ideal of $\mathbb{F}[V]$, i.e. $\bigoplus_{i>0} \mathbb{F}[V]_{i}$.

Definition 1.1. Let $H$ be a graded connected commutative algebra over a field $\mathbb{F}$. We say that $H$ is a Poincaré duality algebra of formal dimension $d$, if

(1) $H_{i}=0$ for all $i>d$,

(2) $\operatorname{dim}_{\mathbb{F}}\left(H_{d}\right)=1$,

(3) the bilinear form $H_{i} \otimes_{\mathbb{F}} H_{d-i} \longrightarrow H_{d}, a \otimes b \mapsto a \cdot b$, is nonsingular, i.e. an element $a \in H_{i}$ is 0 if and only if $a \cdot b=0$ for all $b \in H_{d-i}$.

If $H$ is a Poincaré duality algebra of formal dimension $d$, then there is an element $[H] \neq 0$ in $H_{d}$. Such an element is referred to as a fundamental class for $H$.

Received by the editors June 23, 2003 and, in revised form, January 7, 2005. 2000 Mathematics Subject Classification. Primary 13A50; Secondary 20 F55.

Key words and phrases. Invariant theory, pseudoreflection groups.

(C)2005 American Mathematical Society Reverts to public domain 28 years from publication 
The following theorem is well known.

Theorem 1.2 (G.C. Shephard, J.A. Todd and C. Chevalley). If $\varrho: G \hookrightarrow G L(V)$ is a representation of a finite group $G$ over a field $\mathbb{F}$ and $|G| \in \mathbb{F}^{\times}$, then $\mathbb{F}[V]^{G}$ is a polynomial algebra if and only if $G$ is a pseudoreflection group.

Recall that a linear automorphism $s: V \longrightarrow V$ is called a pseudoreflection if it is not the identity, has finite order, and $\operatorname{dim}_{\mathbb{F}}((1-s) V)=1$. A pseudoreflection group is the group generated by pseudoreflections.

For a field $\mathbb{F}$ of characteristic 0 we have $(8],[16$, or 9$]$ )

Theorem 1.3 (R. Steinberg, R. Kane). If $\varrho: G \hookrightarrow G L(V)$ is a representation of a finite group $G$ over a field $\mathbb{F}$ of characteristic 0 , then $G$ is a pseudoreflection group if and only if $\mathbb{F}[V]_{G}$ is a Poincaré duality algebra.

In this note we shall prove the result of the Theorem (1.3) for the case $|G| \in \mathbb{F}^{\times}$, namely

Main Theorem 1.4. If $\varrho: G \hookrightarrow G L(V)$ is a representation of a finite group $G$ over a field $\mathbb{F}$ and $|G| \in \mathbb{F}^{\times}$, then $G$ is a pseudoreflection group if and only if $\mathbb{F}[V]_{G}$ is a Poincaré duality algebra.

To prove the Main Theorem we make use of a result of L. Smith ([13]).

Theorem 1.5 (L. Smith). Let $\mathbb{F}$ be a field, let $V \cong \mathbb{F}^{n}$, and let $f_{1}, \cdots, f_{n} \in \mathbb{F}[V]$ be a regular sequence of maximal length. Then the quotient $F[V] /\left(f_{1}, \cdots, f_{n}\right)$ is a Poincaré duality algebra.

Corollary 1.6. If $\varrho: G \hookrightarrow G L(V)$ is a representation of a finite group $G$ over a field $\mathbb{F}$ of arbitrary characteristic and $F[V]^{G}$ is a polynomial algebra, then $\mathbb{F}[V]_{G}$ is a Poincaré duality algebra.

Therefore, we only need to prove that the converse of Corollary 1.6 is true in the case $|G| \in \mathbb{F}^{\times}$. In the next two sections, we explain the process.

\section{Preliminaries}

We assume that $\mathbb{F}$ is a perfect field of characteristic $p$. First of all, we recall the construction of the commutative rings, denoted by $W_{m}(\mathbb{F})$, for all $m \geq 1$, with the use of Witt's vector calculus. We call $W_{m}(\mathbb{F})$ the ring of Witt vector of length $m$ over $\mathbb{F}$. The elements in $W_{m}(\mathbb{F})$ are $m$-tupels $\left(a_{1}, \cdots, a_{m}\right), a_{i} \in \mathbb{F}, i=1, \cdots, m$, with a particular ring structure; for more details see [5], [17. These rings define the projective limit $W(\mathbb{F}):=\lim _{r} W_{r}(\mathbb{F})$; the ring $W(\mathbb{F})$ is the ring of Witt vectors of infinite length over $\mathbb{F}$. We refer to $W(\mathbb{F})$ as the Witt-ring of $\mathbb{F}$. The Witt-ring $W(\mathbb{F})$ is a discrete valuation ring whose residue class field is just the field $\mathbb{F}$. The Witt-ring $W(\mathbb{F})$ is an integral domain of characteristic 0 , so that its fraction field, denoted by $\mathbb{K}$, is also of characteristic 0 . If $\mathbb{F}$ is a prime field $\mathbb{F}_{p}$, the Witt-ring $W\left(\mathbb{F}_{p}\right)$ is exactly the $p$-adic integers $\hat{\mathbb{Z}}_{p}$.

The next step is to assume $\varrho: G \hookrightarrow G L(n, \mathbb{F})$ is a representation of $G$ over $\mathbb{F}$. In the sequel we shall define a representation $\tilde{\varrho}: G \hookrightarrow G L(n, W(\mathbb{F}))$ realized as a lifting of $\varrho$ from $\mathbb{F}$ to $W(\mathbb{F})$. Since we have the inclusion $W(\mathbb{F}) \hookrightarrow \mathbb{K}$, the lifting from $\tilde{\varrho}: G \hookrightarrow G L(n, W(\mathbb{F}))$ to $\hat{\varrho}: G \hookrightarrow G L(n, \mathbb{K})$ is obvious. Furthermore, if the ring $\mathbb{F}[V]_{G}$ of coinvariants is a Poincaré duality algebra, so is $W(\mathbb{F})[M]_{\tilde{G}}$ and also $\mathbb{K}[T]_{\hat{G}}$. By Theorems 1.2 and $1.3 \mathbb{K}[T]^{\hat{G}}$ is then a polynomial algebra. As a 
consequence we will show in the next section that $\mathbb{F}[V]^{G}$ is a polynomial algebra as well. In this section, we give the argument for the lifting of a representation $G \hookrightarrow G L(n, \mathbb{F})$ on $W(\mathbb{F})$ and $\mathbb{K}$.

Notation. $M:=W(\mathbb{F})$-module of rank $n$, so that $V \cong \mathbb{F} \otimes_{W(\mathbb{F})} M \cong \mathbb{F}^{n}$,

$T:=\mathbb{K} \otimes M \cong \mathbb{K}^{n}$,

$G:=\varrho(G) \leq G L(n, \mathbb{F}) \cong G L(V)$,

$\tilde{G}:=\tilde{\varrho}(G) \leq G L(n, W(\mathbb{F})) \cong G L(M)$,

$\hat{G}:=\hat{\varrho}(G) \leq G L(n, \mathbb{K}) \cong G L(T)$

Note that $|G|=|\tilde{G}|=|\hat{G}|$.

Theorem 2.1. Suppose that $G$ is a finite group with $|G| \in \mathbb{F}^{\times}$. Let $\rho: G \hookrightarrow$ $G L(n, \mathbb{F})$ be a representation of $G$ over the field $\mathbb{F}$. Then $\varrho$ can be lifted to $\varrho: G \hookrightarrow$ $G L(n, W(\mathbb{F}))$, and this lifting is unique, up to conjugation.

Since $W(\mathbb{F})=\lim _{r} W_{r}(\mathbb{F})$, it follows that $G L(n, W(\mathbb{F}))=\lim _{r} G L\left(n, W_{r}(\mathbb{F})\right)$. It is then enough to prove that:

Theorem 2.2. Suppose that $G$ is a finite group with $|G| \in \mathbb{F}^{\times}$. Let $\varrho_{r}: G \hookrightarrow$ $G L\left(n, W_{r}(\mathbb{F})\right)$ be a representation of $G$ over the ring $W_{r}(\mathbb{F})$. Then $\varrho_{r}$ can be lifted to a representation $\varrho_{r+1}: G \hookrightarrow G L\left(n, W_{r+1}(\mathbb{F})\right)$ for all $r \geq 1, r \in \mathbb{N}$. This lifting is unique, up to conjugation.

The sketch of the proof is as follows: We consider the exact sequence of groups

$$
0 \longrightarrow \operatorname{ker}\left(\breve{\pi}_{r}\right) \hookrightarrow \breve{G} \stackrel{\breve{\pi}_{r}}{\longrightarrow} \varrho_{r}(G) \longrightarrow 1(r \geq 1),
$$

where $\breve{G} \leq G L\left(n, W_{r+1}(\mathbb{F})\right)$ is a subgroup and $\operatorname{ker}\left(\breve{\pi}_{r}\right)$ is an abelian $p$-group and a normal subgroup of $\breve{G}$.

Since $|G| \in \mathbb{F}^{\times}$, the cohomology group $H^{i}\left(\varrho_{r}(G), \operatorname{ker}\left(\breve{\pi}_{r}\right)\right)=0$ for all $i \geq 1$ (see [6]). Since $H^{2}\left(\varrho_{r}(G), \operatorname{ker}\left(\breve{\pi}_{r}\right)\right)=0$ it follows that this exact sequence (1) splits. Thus we can choose a splitting and define $\varrho_{r+1}$. The set of $\operatorname{ker}\left(\breve{\pi}_{r}\right)$ conjugation classes of splitting homomorphisms $\varrho_{r}(G) \longrightarrow \breve{G}$ is in 1-1 correspondence with the first cohomology group $H^{1}\left(\varrho_{r}(G), \operatorname{ker}\left(\breve{\pi}_{r}\right)\right)$. Thus there is precisely one conjugation class.

We have therefore constructed a representation $\tilde{\varrho}: G \hookrightarrow G L(n, W(\mathbb{F}))$ from a given representation $\varrho: G \hookrightarrow G L(n, \mathbb{F})$. Hence, we can define a representation $\hat{\varrho}: G \hookrightarrow G L(n, \mathbb{K})$ via the inclusion $W(\mathbb{F}) \hookrightarrow \mathbb{K}$.

Remark 2.3. If $\mathbb{F}$ is a field of characteristic $p \neq 0$ which is not perfect, then we can always find a local ring $R$ of characteristic 0 whose residue class field is $\mathbb{F}$. The ring $R$ may be taken to be a subring of the Witt $\operatorname{ring} W(\mathbb{F})$ of $\mathbb{F}$ (see [1] ). Therefore, Theorem 2.1 is as well true for the ring $R$. In the next section we work with $R$ instead of $W(\mathbb{F})$, i.e. we set $\mathbb{K}=F F(R)$ and $M \cong R^{n}$.

\section{THE MAIN THEOREM}

In this section we use the result of $\S 2$ to prove the following main theorem.

Theorem 3.1. Let $\varrho: G \hookrightarrow G L(n, \mathbb{F}) \cong G L(V)$ be a representation of a finite group $G$ over a field $\mathbb{F}$ of characteristic p. Suppose that $|G| \in \mathbb{F}^{\times}$. If $\mathbb{F}[V]_{G}$ is a Poincaré duality algebra, then $G$ is a pseudoreflection group. 
Proposition 3.2 (see, e.g. 4]). Suppose that $|G| \in \mathbb{F}^{\times}$. If $\tilde{\varrho}: G \hookrightarrow G L(M)$ is a lifting of the representation $\varrho: G \hookrightarrow G L(V)$ and $\varrho: G \hookrightarrow G L(T)$ is an extension of $\tilde{\varrho}$, then there exist isomorphisms

$$
\mathbb{F} \otimes_{R} R[M]^{\tilde{G}} \cong \mathbb{F}[V]^{G} \quad \text { and } \quad \mathbb{K} \otimes_{R} R[M]^{\tilde{G}} \cong \mathbb{K}[T]^{\hat{G}} .
$$

Proof. We consider the mapping $\psi: \mathbb{F} \otimes_{R} R[M]^{\tilde{G}} \longrightarrow \mathbb{F}[V]^{G}$ given by $a \otimes \tilde{f} \mapsto a \tilde{f}$, for all $\tilde{f} \in R[M]^{\tilde{G}}, a \in \mathbb{F}$. It remains to show that $\psi$ is bijective.

$\psi$ is surjective: For an invariant polynomial $f \in \mathbb{F}[V]^{G}$ there is always a polynomial $\tilde{f} \in R[M]$. Since $|G| \in \mathbb{F}^{\times}$, the Reynolds operation $\pi^{\tilde{G}}(\tilde{f})=\frac{1}{|\tilde{G}|} \sum_{\sigma \in \tilde{G}} \sigma \tilde{f}$, for all $\tilde{f} \in R[M]$, is surjective. This implies that $\psi$ is surjective.

$\psi$ is injective: We choose an invariant polynomial $\tilde{f} \in R[M]^{\tilde{G}}$ so that $\psi(1 \otimes \tilde{f})=$ $0, \overline{\text { i.e., } 1 \otimes \tilde{f} \equiv 0} \bmod p$. So, we may choose an invariant polynomial $\tilde{h} \in R[M]^{\tilde{G}}$ with $\tilde{f}=p \tilde{h}$. This immediately implies that $1 \otimes \tilde{f} \equiv 0 \bmod p$. The remaining case is analogous.

The following corollary is an easy consequence of Proposition 3.2.

Corollary 3.3. Suppose that $|G| \in \mathbb{F}^{\times}$. If $\tilde{\varrho}: G \hookrightarrow G L(M)$ is a lifting of the representation $\varrho: G \hookrightarrow G L(V)$ and $\hat{\varrho}: G \hookrightarrow G L(T)$ is an extension of $\tilde{\varrho}$, then there exist isomorphisms

$$
\mathbb{F} \otimes_{R} R[M]_{\tilde{G}} \cong \mathbb{F}[V]_{G} \quad \text { and } \quad \mathbb{K} \otimes_{R} R[M]_{\tilde{G}} \cong \mathbb{K}[T]_{\hat{G}} .
$$

Let $\mathfrak{R}$ be a graded connected commutative Noetherian ring. For a finitely generated graded $\mathfrak{R}$-module $N$ we define a finitely generated graded $\mathfrak{R}_{0}$-module

$$
\operatorname{Soc}(N):=\{x \in N \mid \bar{\Re} \cdot x=0\},
$$

where $\bar{\Re}$, is denoted as $\bigoplus_{i \geq 1} \Re_{i}$, and call it the socle of $N$.

Theorem 3.4. Let $\mathfrak{R}$ be a graded connected commutative Noetherian ring and let $\mathfrak{q}$ be a $\bar{\Re}$-primary ideal in $\mathfrak{R}$. Then the following conditions are equivalent:

(1) $\operatorname{Soc}(\mathfrak{R} / \mathfrak{q})$ is a finitely generated free $\mathfrak{R} / \overline{\mathfrak{R}}$-module of rank 1 .

(2) The quotient $\mathfrak{R} / \mathfrak{q}$ is a Poincaré duality algebra.

Proof. (1) $\Rightarrow$ (2): We put $\mathfrak{R} / \mathfrak{q}=\bigoplus_{i>0} H_{i}$, where $H_{i}$ is the component of $\mathfrak{R} / \mathfrak{q}$ of degree $i$. There is an integer $d \in \mathbb{N}$ so that $H_{d} \cong \operatorname{Soc}(\mathfrak{R} / \mathfrak{q})$ and $H_{i}=0$ for $i>d$. It remains to show that the pairing $H_{i} \otimes H_{d-i} \longrightarrow H_{d}$ is nonsingular. Since the module $H_{d} \cong \operatorname{Soc}(\mathfrak{R} / \mathfrak{q})$ is of rank 1 , we must have a homogeneous element $a \in \mathfrak{R} / \mathfrak{q}, a \neq 0$, satisfying $H_{d} \subseteq(a) \subset \mathfrak{R} / \mathfrak{q}$. Thus, for every homogeneous element $x \neq 0$ in $H_{d}$ there exists a homogeneous element $b$ in $\mathfrak{R} / \mathfrak{q}$ satisfying $x=a b \neq 0$.

$(2) \Rightarrow(1)$ : This is obvious.

From Theorem 3.4 together with Corollary 3.3 we immediately obtain the following theorem.

Theorem 3.5. The conditions are as in Proposition 3.2 , If $\mathbb{F}[V]_{G}$ is a Poincaré duality algebra of formal dimension d, then $R[M]_{\tilde{G}}$ is a Poincaré duality algebra of formal dimension $d$, and therefore, $\mathbb{K}[T]_{\hat{G}}$ is a Poincaré duality algebra of formal dimension d as well. 
We recall again that if the field $\mathbb{K}$ has characteristic 0 , then $\mathbb{K}[T]_{\hat{G}}$ is a Poincaré duality algebra if and only if $\hat{G}$ is a pseudoreflection group, equivalently, $\mathbb{K}[T]^{\hat{G}}$ is a polynomial algebra.

Theorem 3.6. The conditions are as in Proposition 3.2. If $\mathbb{K}[T]^{\hat{G}}$ is a polynomial algebra, then $R[M]^{\tilde{G}}$ is a polynomial algebra, and therefore, $\mathbb{F}[V]^{G}$ is a polynomial algebra as well.

Proof. Let $\mathbb{K}[T]^{\hat{G}}$ be a polynomial algebra generated by invariant polynomials $f_{1}, \cdots, f_{n}$. From the result of Proposition 3.2 we know that for each generator $f_{i}$, $i=1, \cdots, n$, for $\mathbb{K}[T]^{\hat{G}}$ there is an invariant polynomial, denoted by $\tilde{f}_{i}$ in $R[M]^{\tilde{G}}$. Therefore, $R\left[\tilde{f}_{1}, \cdots, \tilde{f}_{n}\right] \longrightarrow R[M]^{\tilde{G}}$ is a monomorphism. We set $d_{i}=\operatorname{deg}\left(f_{i}\right)$ for each $i=1, \cdots, n$. So we have the Poincaré series

$$
P\left(\mathbb{K}[T]^{\hat{G}}, t\right)=P\left(R[M]^{\tilde{G}}, t\right)=P\left(R\left[\tilde{f}_{1}, \cdots, \tilde{f}_{n}\right], t\right)
$$

and

$$
\begin{aligned}
\operatorname{deg}\left(\mathbb{K}[T]^{\hat{G}}\right)= & \frac{1}{|\hat{G}|}=\frac{1}{d_{1} \cdots d_{n}}=\left.(1-t)^{n} P\left(R\left[\tilde{f}_{1}, \cdots, \tilde{f}_{n}\right], t\right)\right|_{t=1} \\
& =\left.(1-t)^{n} P\left(R[M]^{\tilde{G}}, t\right)\right|_{t=1}=\operatorname{deg}\left(R[M]^{\tilde{G}}\right)=\frac{1}{|\tilde{G}|} .
\end{aligned}
$$

Hence, we have $|\hat{G}|=d_{1} \cdots d_{n}=|\tilde{G}|$. In addition, since $\tilde{f}_{1}, \cdots, \tilde{f}_{n}$ are algebraically independent over $R$, we obtain $R[M]^{\tilde{G}} \cong R\left[\tilde{f}_{1}, \cdots, \tilde{f}_{n}\right]$. The proof for the last result is analogous.

From Theorem 3.5 and Theorem 3.6 together with Theorem 1.2 we complete the proof of the main theorem.

Remark 3.7. This result may fail for $p$-groups. There is a counterexample when $p=2$ and $\operatorname{dim}_{\mathbb{F}_{p}} V=4$ (see [15]).

\section{ACKNOWLEDGMENTS}

I am very grateful to professor Larry Smith for his support in providing his precise suggestions in our discussions.

\section{REFERENCES}

1. J.F. Adams and C.W. Wilkerson, Finite H-Spaces and Algebras over the Steenrod Algebra, Annals of Math. 111(1)(1980), 95-143. MR0558398 (81h:55006)

2. D.J. Benson, Polynomial Invariants of Finite Groups, Cambridge Univ. Press, London, 1993. MR.1249931 (94j:13003)

3. K.S. Brown, Cohomology of Groups, GTM 87, Springer-Verlag, New York, 1982. MR0672956 (83k:20002)

4. A. Clark and J. Ewing, The Realization of Polynomial Algebras as Cohomology Rings, Pac. J. Math. 50(1974), 425-434. MR0367979 (51:4221)

5. H. Hasse, Zahlentheorie, Akademie-Verlag, Berlin, 1963. MR0153659 (27:3621)

6. P.J. Hilton and U. Stammbach, A Course in Homological Algebra, GTM 4, Springer-Verlag, New York, 1997. MR1438546 (97k:18001)

7. N. Jacobson, Basic Algebra II, W.H. Freeman and Company, New York, 1989. MR.1009787 (90m:00007)

8. R. Kane, Poincaré Duality and the Ring of Coinvariants, Canad. Math. Bull. 37(1)(1994), 82-88. MR 1261561 (96e:51016) 
9. R. Kane, Reflection Groups and Invariant Theory, CMS Books in Math., Springer-Verlag, New York, 2001. MR1838580(2002c:20061)

10. T.-C. Lin, Über Poincarédualitätsalgebra in der Invariantentheorie, Doktorarbeit, University of Göttingen, 2003. MR2015573 (2004i:13005)

11. C. Schoeller, Groupes Affines, Commutatifs, Unipotents sur un Corps Non Parfait, Bull. Soc. Math. France 100(1972), 241-300. MR0393052(52:13863)

12. J.-P. Serre, Linear Representations of Finite Groups, GTM 42, Springer-Verlag, New York, 1977. MR0450380 (56:8675)

13. L. Smith, A Note on the Realization of Complete Intersection Algebras by the Cohomology of a Space, Quat. J. of Math 33(2)(1982), 379-384. MR0668184 (84a:57044a)

14. L. Smith, Polynomial Invariants of Finite Groups, A.K. Peter-Verlag, Wellesley, Massachusetts, 1995. MR.1328644 (96f:13008)

15. L. Smith, On a Theorem of R. Steinberg on Rings of Coinvariants, Proc. of the Amer. Math. Soc. 131(4)(2002), 1043-1048. MR.1948093 (2003k:13008)

16. R. Steinberg, Differental Equations Invariant under Finite Reflection Groups, Trans. Amer. Math. Soc. 112(1964), 392-400. MR0167535 (29:4807)

17. E. Witt, Zyklische Körper und Algebren der Charakteristik p vom Grade $p^{n}$, J. Reine Angew. Math. 176(1936), 126-140.

Mathematisches Institut, Georg-August-Universität Göttingen, Bunsenstrasse 3-5, D-37073 Göttingen, Germany - and - Department of Applied Mathematics, Feng Chia University, 100 Wenhwa Road, Taichung 407, Taiwan, Republic of China

E-mail address: lintc@fcu.edu.tw 